\title{
Design an Adjustable Narrow Correlator to Track GPS Signals
}

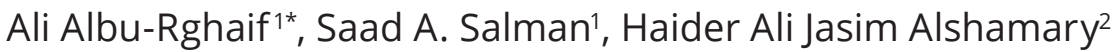 \\ 1 Department of Computer Engineering, College of Engineering, University of Diyala, \\ Mail Box No. 1, College of Engineering Branch, Ba'quba Mail Office, Diyala, Iraq \\ 2 Department of Communication Engineering, College of Engineering, University of Diyala, \\ Mail Box No. 1, College of Engineering Branch, Ba'quba Mail Office, Diyala, Iraq \\ *Corresponding author, e-mail: ali.alburghaif@yahoo.com
}

Received: 08 December 2018, Accepted: 09 April 2019, Published online: 09 July 2019

\begin{abstract}
Multipath signals problem is still the main challenge for the global positioning system (GPS) receiver to obtain an accurate localization measurement. However, many techniques have been developed to mitigate the impact of these undesired signals at the expense of adding a burden to the GPS receiver design. Even though, none of the techniques in the works of literature was able to solve the loose signal lock issue efficiently. In this piece of work, we used a new approach to maintain the tracking synchronization when the receiver moves from outdoors to indoors /harsh environment. We design a dynamic narrow correlator in the delay lock loop, which made the space between the early and late correlators adjusted according to the receiver's location. Consequently, this will enable the receiver to keep tracking the received GPS signal in a challenging environment and reduce the thrashing the available resources (power/ processing) to acquire the GPS signal again. The results show that the ranging error has been reduced in comparison with a traditional narrow correlator, as well as the bit transition in the in-phase arm has been also maintained.
\end{abstract}

Keywords

tracking GPS signal, adjustable narrow correlator, early-late space

\section{Introduction}

The signal processing of the GPS signal, at the user end, can be divided into four stages. Firstly, the RF front-end stage that responsible for receiving and digitizing the GPS signal. Acquiring stage, in turn, detects the visible satellite signals and provides rough values of code phase and carrier with a Doppler frequency of the GPS signals. Next, the tracking stage is responsible to refine these code and carrier values that were delivered from the previous stage, as well as, to keep the synchronization with GPS satellites. Eventually, the demodulation stage extracts the navigation data of the acquired GPS satellites and computes the GPS receiver position [1].

In this software oriented work, we focus on tracking GPS signal, tracking stage, which mainly comprises two stages, the phase lock loop and the delay lock loop. The phase lock loop is utilized to track the carrier frequency while the delay lock loop is responsible for tracking the specific code phase in the acquired signal. The ultimate delay lock loop performance is essentially challenged by the multipath signals which are reflected from interference of original GPS signal.
Several solutions have been developed to enhance the functionality of tracking GPS signals and to mitigate the multipath effect. However, most of these solutions added more complexity to the receiver design in the sense of increasing size, processing resources and power consumption. Using antenna array or increasing the code phase resolution by decrease the space between the early and late correlators, or by adding another pair of correlators were some of proposed solutions [2, 3]. Even though, correspondence past works have mitigated undesired signal but, at the same time, decrease the signal to nice ration and hence system performance [4]. In principle, the undesirable influence of multipath signals is clearly highlighted as a major drawback when the GPS receiver located in a kinematic environment, i.e. moving for outdoors to indoors or vice versa. In this case the acquiring signal should be processed again, which leads to thrash the available resources. To maintain an efficient performance and overcome software receivers' problems, we have designed tracking GPS signal based on an adjustable narrow correlator (ANC). In this work the space between the early and late correlators 
will vary according to the measurement error, which produces a high code resolution in outdoors and maintain the synchronization in indoors without the necessity to perform the acquisition once more again.

The paper is organized as follows. Section 2 summarizes previous tracking techniques. Section 3 describes the implementation of our proposed design. The performance results of our ANC are discussed in comparison with static design in Section 4. Finally, the conclusions are listed in Section 5.

\section{Previous tracking techniques}

The increasing demand for the positioning solution in portable devices (smartphones, tablet, etc.) and their applications, lead to develop various practical localization techniques. This section will try to cover the most common techniques that offer a multipath mitigation in order to enhance the localization accuracy.

Narrow correlator which was introduced to GPS receiver by NovAtel communications Ltd., is the well know techniques to reduce the impact of multipath [5]. Using 10 channels to track GPS signal with different early-late space with different discriminator in order to mitigate as much as possible of the multipath signal error. The narrow correlator technique replaced the (1 chip) space of standard receiver with ( 0.1 chip) between the early and late correlators which are using in the DLL for GPS tracking. Also, the researchers proved that the multipath signal error is directly proportional to the early-late space. In other words, the reduction of multipath depends on the amount of spacing (i.e. the reduction of multipath signal will increase when the early-late space is small).

Because the narrowing space techniques was so effective dealing with multipath signal problem, many more approaches were introduced in the literatures based on it. [6], for example, introduces the reduction of multipath error in L1 GPS by taking the advantage of narrow correlator spacing design to derive the early-late slope technique. The technique in [5] depends on tracking error, where the error will equal to zero when the early and late correlators are placed on the same level on each side while when there is an error (the early and late correlator not on the same level) it feeds back to the hardware that makes both of the early and late correlators centered on the peak.

On other hand, [7] develops narrow correlator at Septentrio satellite navigation for short delay multipath mitigation. The main concept of this work focused on estimating the multipath error through adding another correlator to the late correlator side, where the output of the added correlator is independent and not affected the tracking process. This method achieved up to $50 \%$ more reduction in-phase multipath comparted with the standard techniques (narrow correlator). NovAtel communications Ltd in turn, added another pairs of correlators with the early and late correlators to produce pulse aperture correlator to achieve more mitigation to multipath signal [8]. Similarly, [9] introduced double delta correlator with the advantage of rejecting the multipath by eliminating the shorter delays.

The principles of the vision correlator was illustrated by [10], based on hardware implementation. Vision correlator measures the chip transition (from 0 to 1 or 1 to 0 ) from received signal, in meaning, the hardware processor filter transitions over period of time to estimate the line of sight and multipath information (code delay, power and carrier phase). This technique is able to reduce the multipath signal error when the delayed signal (multipath) is reflected from less than $10 \mathrm{~m}$.

In addition, [11] adapted a different direction of analysis in mitigation multipath signals since it concerns about modifying the carrier phase rather than C/A code in the aforementioned works. The difference between the early and late carrier phase is calculated to keep maximum energy in the in-phase channel and thus make the GPS receiver sensitive in the presence of the multipath not only for the code phase but with the carrier phase that would be relatives to the phase difference.

A multipath estimating delay lock loop (MEDLL) was designed to measure the multipath parameters [12]. The MEDLL approach depends on estimating the values of code phase, carrier phase and the power for multipath signal by using a maximum likelihood algorithm. The advantages of the multipath meter are useful for static environment and more sense to the long delay multipath due to estimating parameters (power, delay and phase). However, this meter is not efficient for dynamic environment and low sense to the short delay multipath signal. In the same vein, a new approach was designed to mitigate the multipath signals, which called reduced search space maximum likelihood (RSSML) delay estimator [13]. The RSSML consists of five steps. Firstly, the noise estimation step, where the noise is estimated by calculating the mean out-of-1-chip values at the early side from the prompt correlator. Secondly, the competitive peak generation step, which depends on the noise estimation to generate peaks, and on only one computed peak is above the threshold. This will reduce the searching from large number of correlators to few competitive delay applicants. 
Then, the reference correlation functions reading and translation step. At the beginning of this step the reference correlation functions are read, and then it translated to the matching applicant delay. Next, the minimum mean square error (MMSE) is calculated for each candidate delay index. Finally, the line of sight (LOS) delay estimation step, which chooses the lowest MMSE. However, this approach needs large number of correlation for channel estimation to mitigate the multipath.

A dynamic algorithm to track GNSS signals was designed, based on Kalman filter (KF), using adaptive four-state KF-based [14]. The four states are delay lock loop (DLL), frequency lock loop (FLL), and phase lock loop (PLL) filters with a combined adaptive Kalman filter. This algorithm is assisted by an inertial navigation system (INS) to overcome losing lock of the PLL. The authors show that using of the adaptive KF will produce more accurate estimation for the Doppler frequency, as well as reduce the measurement error.

\section{Our ANC structure}

After acquiring the GPS signals, the coarse values of code phase delay and carrier frequency will be delivered to DLL and PLL respectively. The DLL is implemented as a normal narrow correlator which generates three replicas of codes. These codes are called early (E), prompt (P) and late (L) with space of chip delay, i.e. $€$ generated with chip delay, (L) with chip delay and (P) at 0 chip delay, as shown in Fig. 1. Then, the acquired signal is multiplied with the three replicas to determine the highest correlation. Next, according to the highest correlation the code phase will be rearranged (increased if the highest correlation in the $\mathrm{E}$ or decreased if the highest correlation in the L). The idea of this process is to make as much as possible the highest peak/correlation in the middle $(\mathrm{P})$. However, the presences of multipath, in indoors or urban-canyons environment extends this process to be obtained in the $\mathrm{P}$ correlator. Consequently, the space between $\mathrm{E}$ and $\mathrm{L}$ correlators

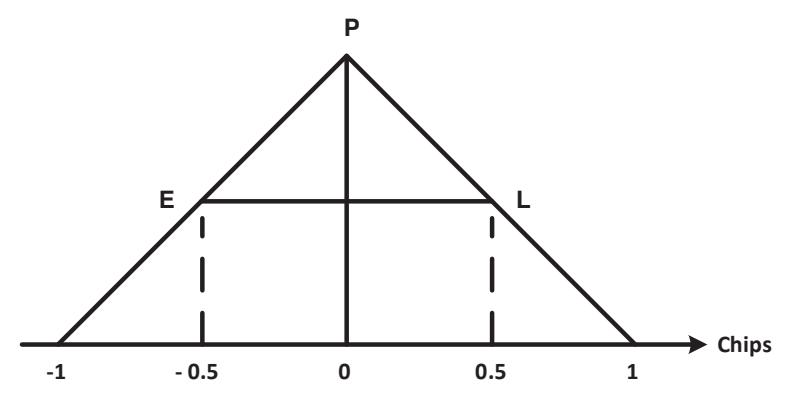

Fig. 1 Traditional E-P code tracking determines the code phase resolution and the amount of multipath delay [15], as in Eq. (1)

$\operatorname{Max}_{\text {delay }}=1+D / 2$

where " 1 " is equal to one chip and " $D$ " represents the space between $\mathrm{E}$ and L.

Therefore, a dynamic solution is the most appropriate to have high code phase resolution in outdoors, and at the same time to keep tracking of the signal in indoor environment without lose signal lock. The idea here in our approach is to make the space or the distance between $\mathrm{E}$ and $\mathrm{L}$ adjusted (move up or down) based on the measurement error, as shown in Fig. 2. In the implementation of our proposed system, the new space between $E$ and $L$ is determined according to the output value of the code discriminator, we illustrate this in Fig. 3.

We can see in Eq. (2) that, the utilized code discriminator in this work is a non-coherent normalized early minus late discriminator. The main advantage of such discriminator type is able to maintain tracking noisy or indoor signals, as well as outdoor signals [1].

Code Error $=\frac{\left(I_{E}^{2}+Q_{E}^{2}\right)-\left(I_{L}^{2}+Q_{L}^{2}\right)}{\left(I_{E}^{2}+Q_{E}^{2}\right)+\left(I_{L}^{2}+Q_{L}^{2}\right)}$.

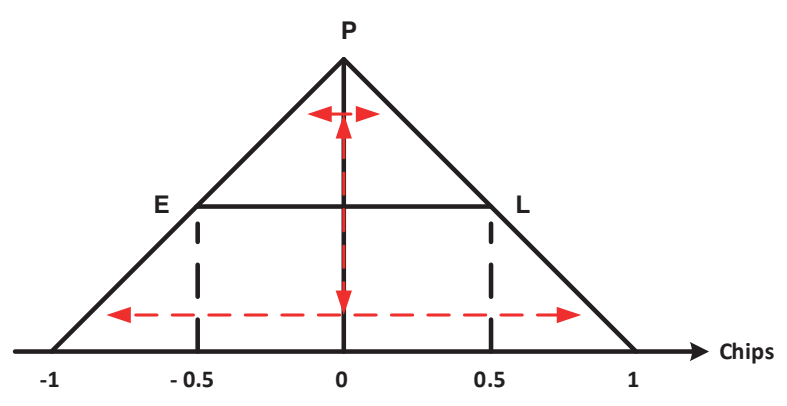

Fig. 2 Adjustable E-L code tracking

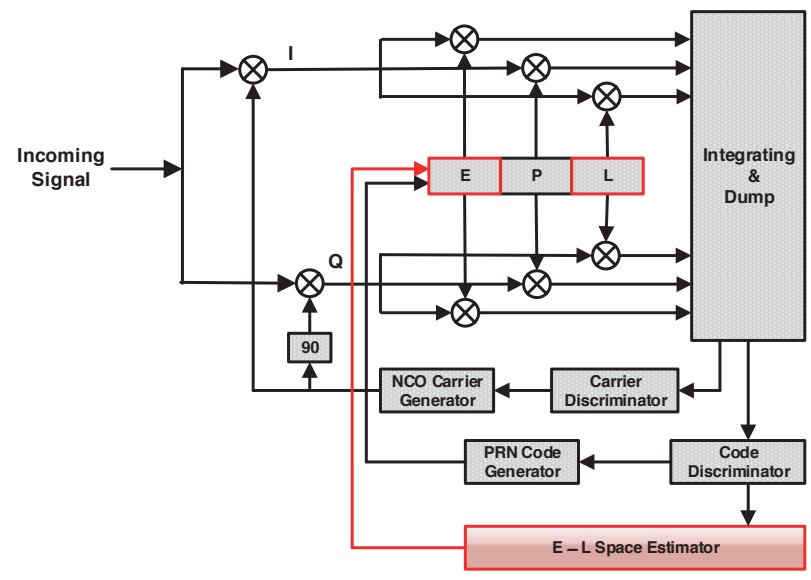

Fig. 3 Adjustable tracking block diagram 
Where code error represents the discriminator output, $I_{E}$ and $I_{L}$ are the in-phase $(I)$ of early and late correlators respectively; $Q_{E}$ and $Q_{L}$ are the quadrature-phase $(Q)$ of early and late correlators respectively.

In this work, four levels of E-L space are proposed and started from 0.1 chip to 0.9 chip. The value of each level is proportional to the correlation output from I and Q arms in the early and late correlators (see Eq. (2)). Thus, the new space depends on the ratio of the early (I\&Q) arm to the late (I\&Q) arm (raw DLL discriminator) and the new measured code error (filtered DLL discriminator), i.e. these error and ratio measurement reflects if the GPS receiver in outdoors or indoors environment. Then these values are compared with a certain threshold to decide whether a space should be moving up or down with a moving step of 0.2 chip. It is worthwhile to mention that the resolution of the chip delay depends on the sampling frequency (how many samples in one chip).

\section{Our ANC results and analysis}

In our experiments, a fixed or static E-L space with 0.5 chip is employed for the comparison in terms of discriminator outputs (code error), bit transition and bit error rate. The GPS signals that used in our experiments are obtained from [1], and it is called "GPS data-Discrete Componentsfs38_192-if9_55.bin" as outdoors signals, and each chip is represented by around 37 samples. While, the indoors GPS signals are modified by using white Gaussian noise to simulate urban-canyons environment. Therefore, in order to evaluate the performance of our new approach, comparisons with various analyses have been utilized with different GPS signals scenarios. The first scenario represents the strong signal in outdoors whereas the second scenario is the weak signal when moving the GPS receiver into indoors, with 3 sec of the received signals length in all experiments.

\subsection{Outdoors results}

The acquired signals from satellites (sv15 and sv22) are chosen in outdoors comparison to illustrate how our solution is better than the static one in terms of bit transition and code error.

The discrete time scatter, as depicted in Fig. 4 and Fig. 5 clearly shows that our solution has maintained the bit transition in the In-phase arm. Thus, it will help to sync with the received GPS signal and then to extract the navigation message from the acquired satellite. Also, the range of the discriminator output that represents the normalized code error is reduced (from -3 to -1.5 in Fig. 6
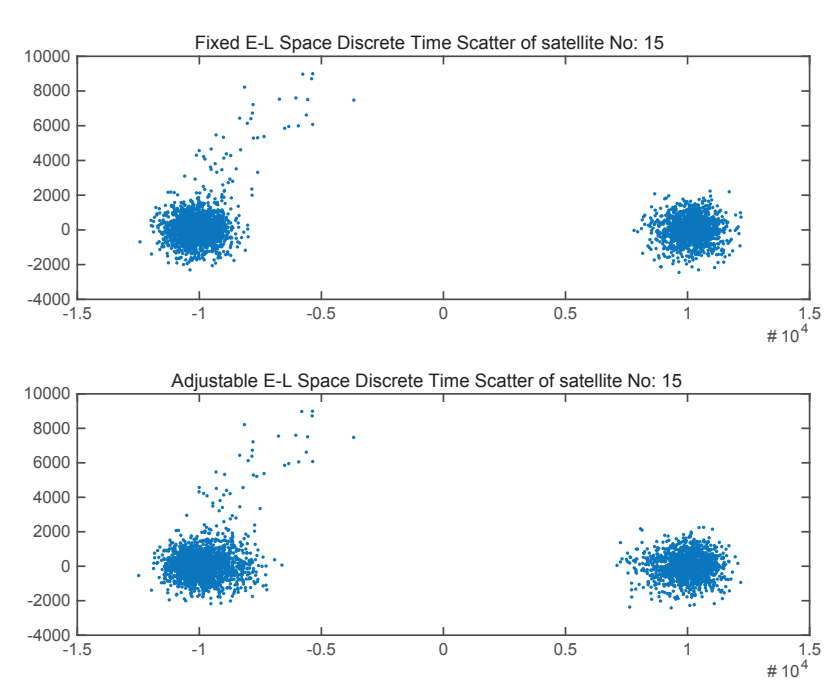

Fig. 4 Bit transition in outdoors of satellite 15
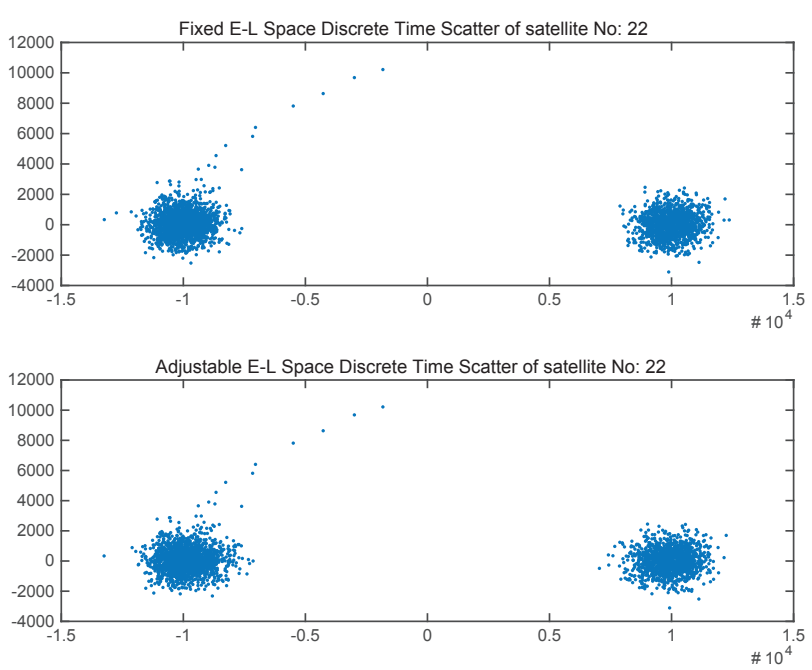

Fig. 5 Bit transition in outdoors of satellite 22
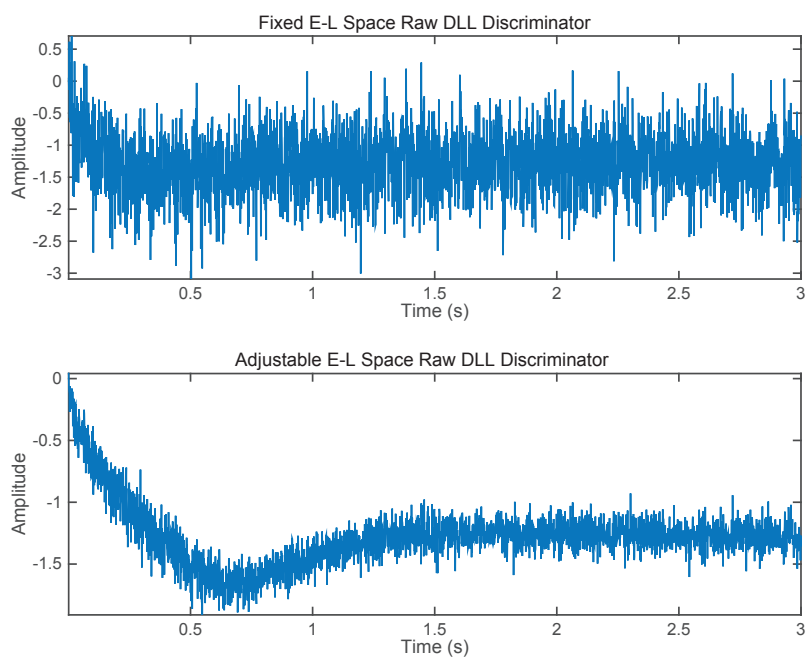

Fig. 6 Normalized raw DLL discriminator in outdoors of satellite 15 
and Fig. 7) for both satellites 15 and 22. Eventually, the trend of the filtered DLL discriminator is close to zero, as shown in Fig. 8 and Fig. 9.

\subsection{Indoors results}

In order to simulate the indoors/multipath signals, we have added a white Gaussian noise to the same outdoors signals, such that the signal to noise ratio is equals to $-10 \mathrm{~dB}$. Same analyzing has been utilized as the outdoors experiments in terms of bit transition, normalized code error and numerically controlled oscillator.

\subsubsection{Bit transition}

Our solution has overcome the loss of the received signals by increasing the space between the early and late correlator in order to accommodate the desired signal. If we
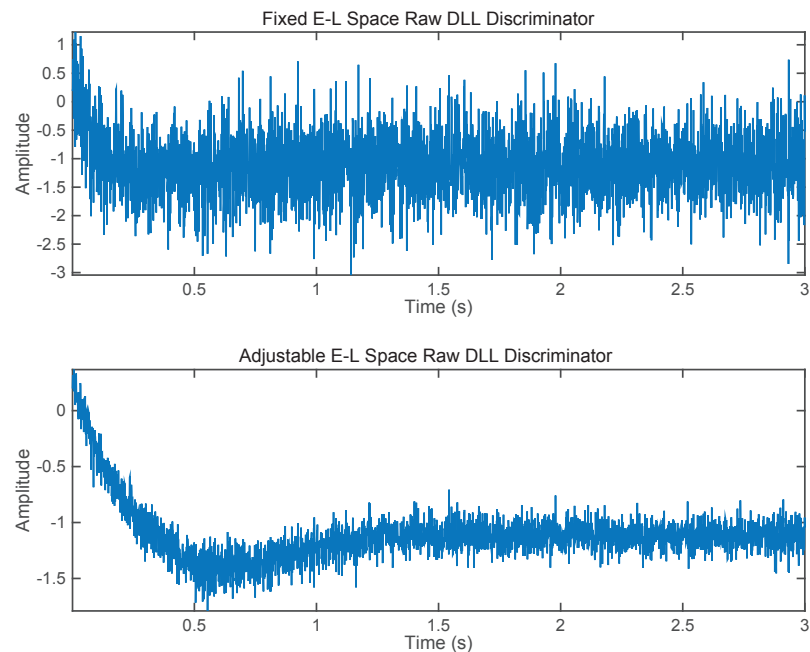

Fig. 7 Normalized raw DLL discriminator in outdoors of satellite 22
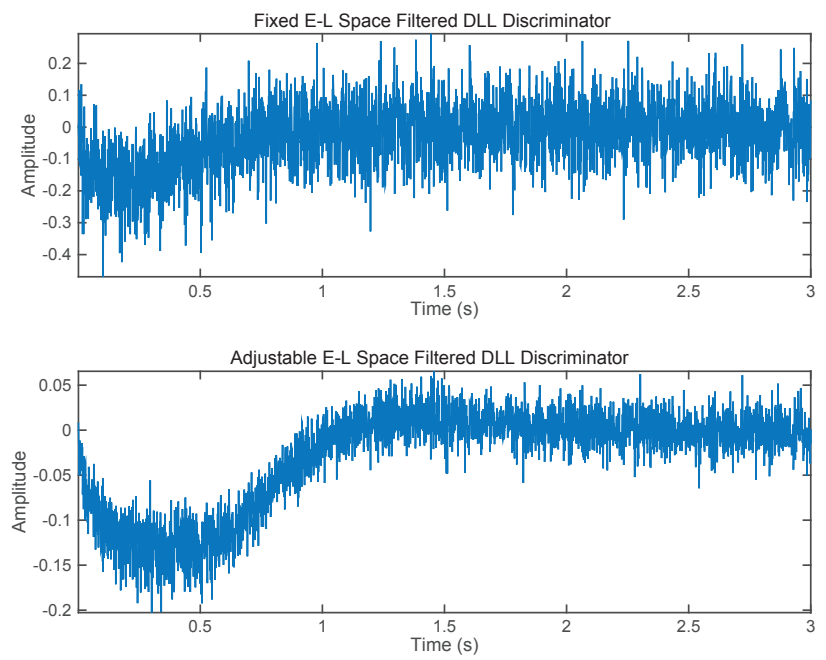

Fig. 8 Filtered DLL discriminator in outdoors of satellite 15 compare the distortion in the bit transition, it's clearly seen that our solution has better performance than the traditional implementation by maintaining the separation in the In-phase arm as shown in (Fig. 10 and Fig. 11). Moreover, our solution shows same performance as outdoor scenarios.

\subsubsection{Normalized code error}

As shown in Eq. (2), the discriminator output represents a normalized code error, which shows the difference between the early and late correlators. Fig. 12 and Fig.13, illustrate how our ANC reduced these measurements to overcome the multipath effect by making both early and late correlators as much as possible in the same level. Below are the summarized of above figures and as follows:

- Fig. 12 the range error of satellite 15 is reduced from $(4,-6)$ to $(0,-2)$ in indoors scenario.
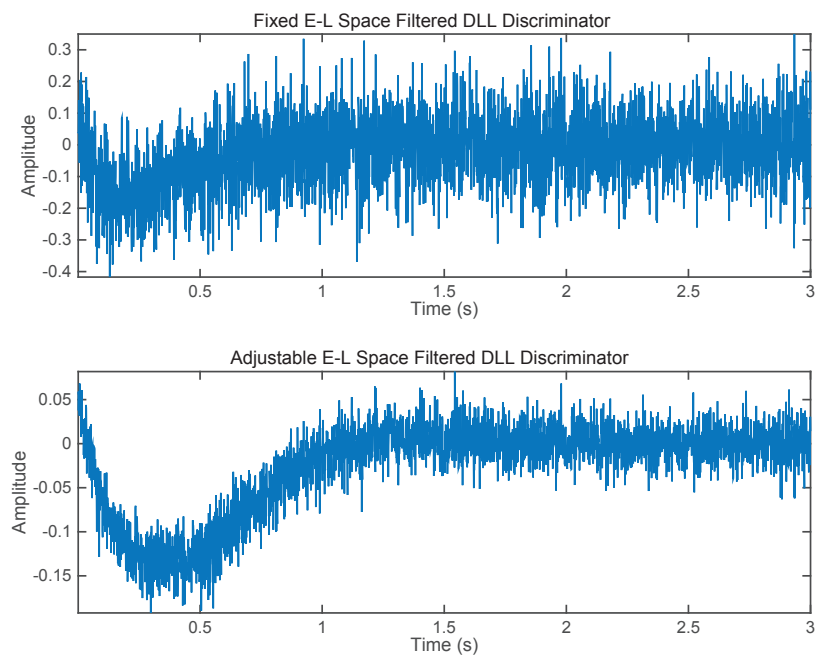

Fig. 9 Filtered DLL discriminator in outdoors of satellite 22
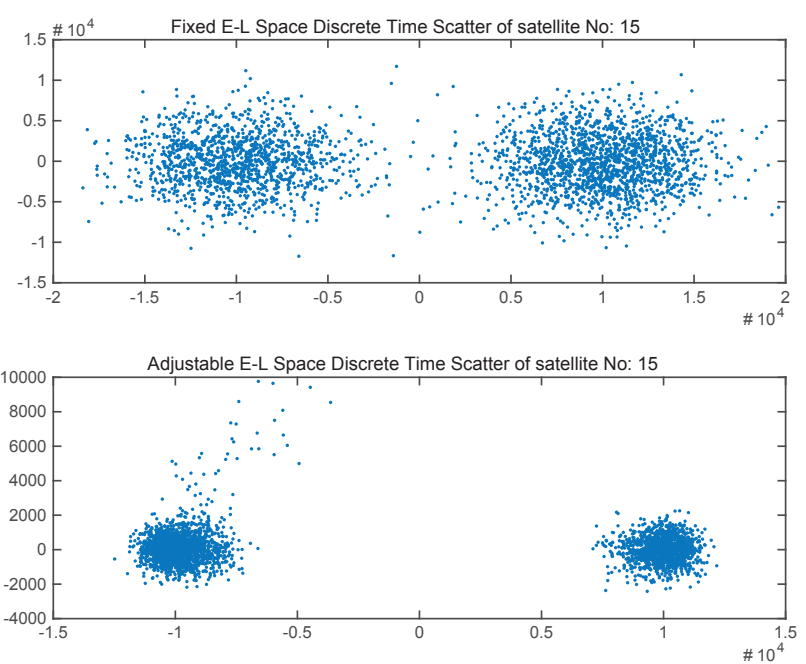

Fig. 10 Bit transition in indoors of satellite 15 
- Fig. 13 the range error of satellite 22 is reduced from $(4,-6)$ to $(0,-1.5)$ in indoors scenario.

\subsubsection{Numerically controlled oscillator (Code NCO)}

In order to maintain the synchronization with the received signal, the discriminator outputs are then filtered based on code loop coefficients (DLL noise bandwidth and DLL damping ratio). This will correct the early, prompt and late correlators, and helps to reduce the code error. As shown in Fig. 14 and Fig. 15, the trend of the filtered DLL discriminator is almost same as outdoors performance and closed to zero as illustrated below:

- Fig. 14 , the range of code NCO reduced from $(0.8$, $-0.8)$ to $(0.05,-0.2)$ in indoors scenario.

- Fig. 15 , the range of code NCO reduced from $(0.8$, $-0.8)$ to $(015,-0.15)$ in indoors scenario.
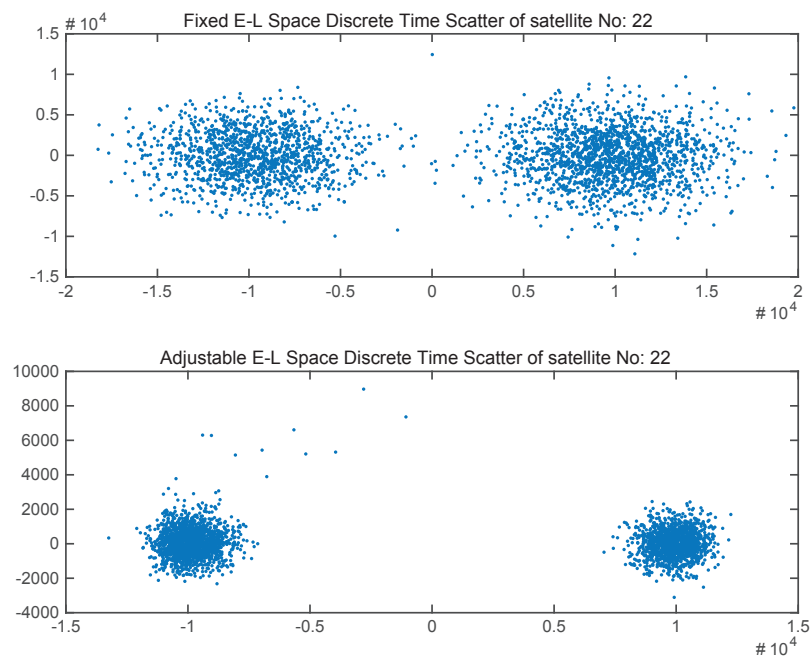

Fig. 11 Bit transition in indoors of satellite 22
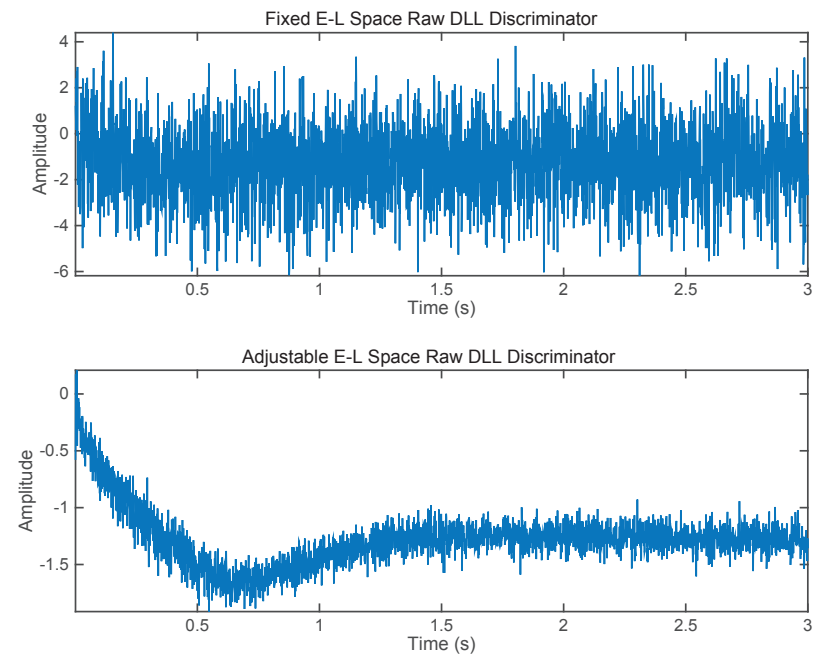

Fig. 12 Normalized raw DLL discriminator in indoors of satellite 15
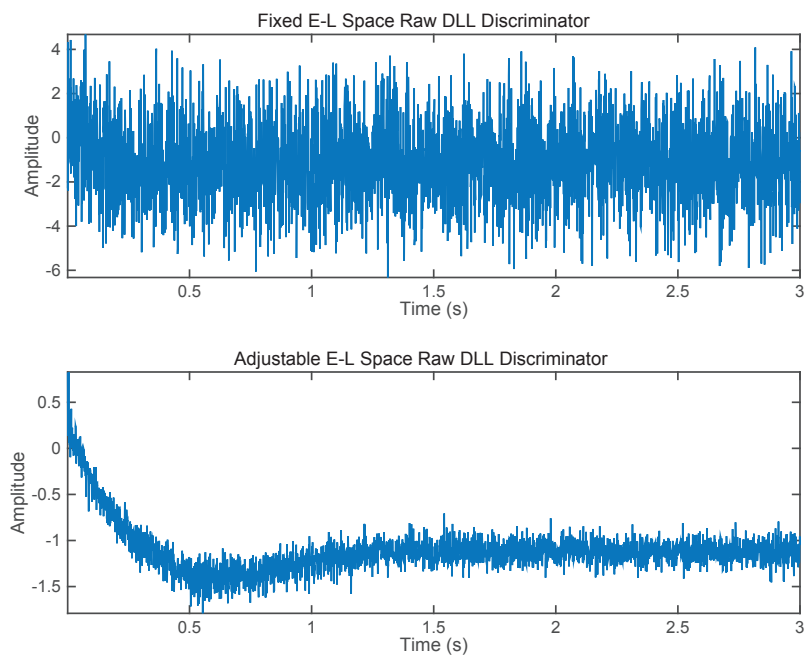

Fig. 13 Normalized raw DLL discriminator in indoors of satellite 22
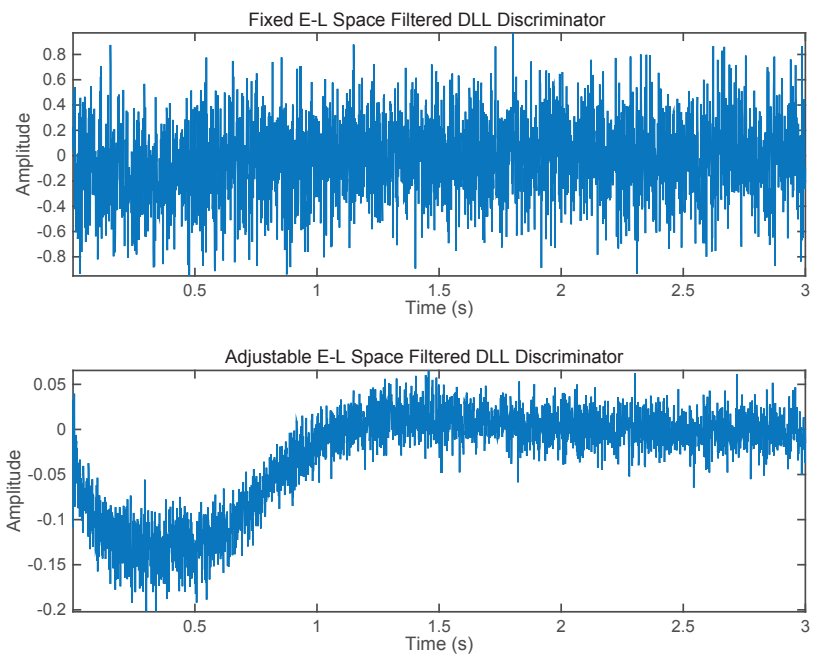

Fig. 14 Filtered DLL discriminator in indoors of satellite 15
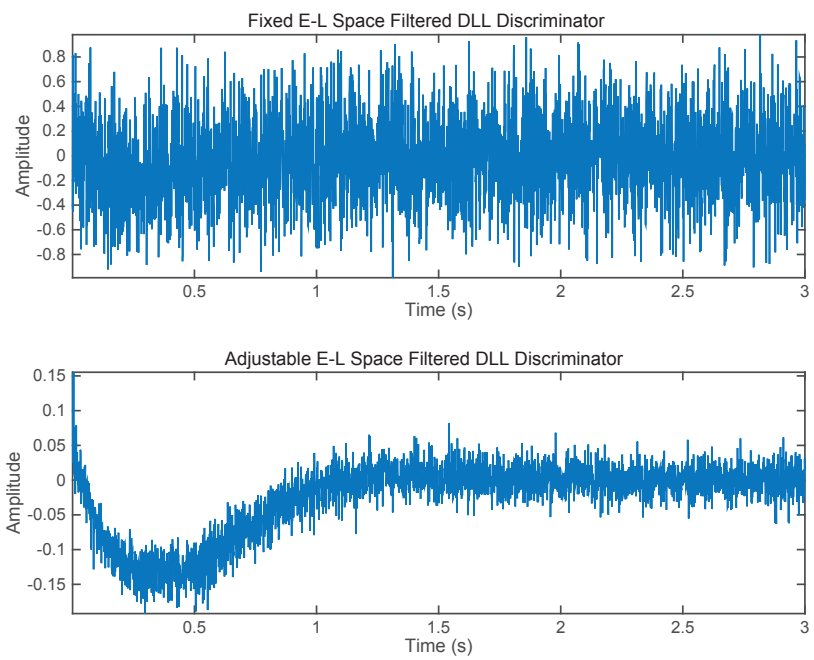

Fig. 15 Filtered DLL discriminator in indoors of satellite 22 


\subsection{Bit error rate results}

It is worthwhile to mention that our analysis has also used the bit error rate (BER) of decoded message in order to measure the validity of our solution. Compared with traditional solution (narrow correlator with 0.5 chip space between early and late correlator), our ACN achieves noticeable higher SNR rate. From Fig. 16, when the BER is, the $\mathrm{ACN}$ achieves about $2 \mathrm{~dB}$ gains over the traditional receivers. This $\mathrm{dB}$ gain was earned because the design of our tracking system has the ability to adjust the E-Lspace according to the location of the GPS receiver, which reduces the code error to the most possible rate.

\subsection{Computational complexity and restriction}

As shown from Fig. 3, the additional cost that added to our ANC is the estimation of E-L space. This operation will require $O(5)$, where $\mathrm{N}$ represents the number of (one) error code comparison and (four) checking to select which level of the new E-L space. On the other hand, the restriction in this work is the amount of allowed indoors signal; where according to Eq. (1) the maximum delay is around 1.5 chip delay. Actually, this amount is equal to the space that has been used before introducing the narrow correlator, and it also enables the GPS receiver to track signal without re-acquiring the signal again.

\section{Conclusions}

In this work, a dynamic solution to track GPS signal has been designed. Our ANC enables the DLL to adjust the

\section{References}

[1] Borre, K., Akos, D. M., Bertelsen. N., Rinder. P., Jensen. S. "A software defined GPS and Galileo receiver: a single-frequency approach", Springer Science \& Business Media, New York, USA, 2007.

https://doi.org/10.1007/978-0-8176-4540-3

[2] Groves, P. D. "Principles of GNSS, inertial, and multisensor integrated navigation systems, 2nd edition [Book review]", Aerospace and Electronic Systems Magazine, 30(2), pp. 26-27, 2015. https://doi.org/10.1109/MAES.2014.14110

[3] Ray, J. K. "Mitigation of GPS code and carrier phase multipath effects using a multi-antenna system", Doctor of Philosophy, The University of Calgary, 2000.

[4] Pratt, A. R. "Performance of Multi-Path Mitigation Techniques at Low Signal to Noise Ratios", In: Proceedings of the 17th International Technical Meeting of the Satellite Division of The Institute of Navigation (ION GNSS 2004), Long Beach, CA, USA, 2004, pp. 43-53.

[5] Dierendonck, A. V., Fenton, P., Ford, T. "Theory and performance of narrow correlator spacing in a GPS receiver", Navigation, 39(3), pp. 265-283, 1992.

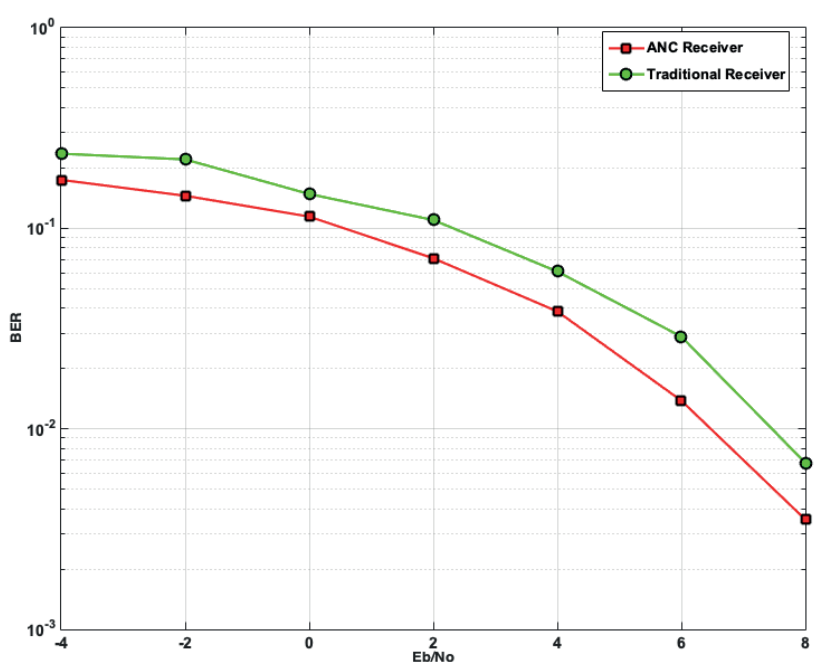

Fig. 16 Bit error rate versus energy per bit to noise power spectral

space between early and late correlators according to the position of the GPS receiver. Our solution performance is analyzed and compared with other standard narrow solution in terms of bit transition, code error, bit error rate and complexity. The results showed that our solution has the ability to mitigate the multipath signal in outdoors and accommodate the GPS signal in indoors.

In conclusion, our literature survey showed that previous solutions have been effectively eliminating the multipath signal. Nevertheless, most of them are designed for static environment, i.e. in urban-canyons scenario. While our ANC solution has been proposed for a dynamic scenario, i.e. when moving from outdoors to indoors or vice versa.

[6] Townsend, B., Fenton, P. "A practical approach to the reduction of pseudorange multipath errors in a L1 GPS receiver", In: Proceedings of the 7th International Technical Meeting of the Satellite Division of the Institute of Navigation, Salt Lake City, UT, USA, 1994.

[7] Sleewaegen, J., Boon, F., "Mitigating short-delay multipath: A promising new technique", In: Proceedings of the 14th International Technical Meeting of the Satellite Division of The Institute of Navigation (ION GPS 2001), Salt Lake City, UT, USA, 2001, pp. 204-213.

[8] Fenton, P., Smith, B., Jones, J. "Theory and performance of the pulse aperture correlator", Technical Report, NovAtel, Calgary, Alberta, Canada, 2004.

[9] McGraw, G., Braasch, M. "GNSS multipath mitigation using gated and high resolution correlator concepts", In: Proceeding National Technical Meeting of the Satellite Division of the Institute of Navigation, San Diego, CA, USA, 1999, pp. 333-342.

[10] Fenton, P., Jones, J. "The theory and performance of NovAtel Inc.'s Vision Correlator", In: Proceedings of the Institute of Navigation (ION GNSS 2005), Long Beach, CA, USA, 2005, pp. 2178-2186. 
[11] Mubarak, O. "Analysis of early late phase for multipath mitigation", In: 21st International Technical Meeting of the Satellite Division of the US Institute of Navigation, Savannah, Georgia, USA, 2008, pp. 669-678.

[12] Townsend, B., Wiebe, J., Jakab, A. "Results and analysis of using the MEDLL receiver as a multipath meter", In: Proceedings National Technical Meeting of the Institute of Navigation, Anaheim, CA, USA, 2000, pp. 73-79.

[13] Bhuiyan, M. Z. H., Lohan, E. S. "Advanced multipath mitigation techniques for satellite-based positioning applications", International Journal of Navigation and Observation, 2010, Article ID: 412393, 2010.

https://doi.org/10.1155/2010/412393
[14] Jing, S., Zhan, X., Liu, B., Chen. M. "Weak and Dynamic GNSS Signal Tracking Strategies for Flight Missions in the Space Service Volume", Sensors, 16(9), Article ID: 1412, 2016. https://doi.org/10.3390/s16091412

[15] Li. L., Zhou, W., Tan, S. "Tracking accuracy of narrow correlator spacing GPS receiver", In: 8th International Conference on Signal Processing, Beijing, China, 2006.

https://doi.org/10.1109/ICOSP.2006.346123 